\title{
Vortex pinning by cylindrical defects in type-II superconductors: Numerical solutions to the Ginzburg-Landau equations
}

\author{
S. M. Maurer, N.-C. Yeh, and T. A. Tombrello \\ Department of Physics, California Institute of Technology, Pasadena, California 91125 \\ (Received 15 January 1996; revised manuscript received 10 July 1996)
}

\begin{abstract}
We numerically integrate the one-dimensional, cylindrically symmetric Ginzburg-Landau equations to calculate the spatial variation of the order parameter and supercurrents for a vortex trapped by a cylindrical defect. We use the resulting field distributions to estimate the pinning energy, and make use of the vortex/twodimensional boson analogy to calculate the depinning temperature. The microscopic behavior of the fields depends on the size, and the conductivity of the cylindrical defect appears to be important for the pinning. [S0163-1829(96)09045-5]
\end{abstract}

\section{INTRODUCTION}

Inhibiting the motion of vortices in type-II superconductors presents a complex physical problem with a significant technological motivation, since thermally-induced vortex motion severely limits the use of high- $T_{c}$ superconductors in practical applications. One solution to the problem, the use of pinning, also opens a new chapter in the thermodynamics of vortices. Columnar defects are now widely used to trap vortices and to increase the current carrying capacity of high- $T_{c}$ superconductors. ${ }^{1,2}$ Yet, except for some phenomenological descriptions, ${ }^{3,4}$ the microscopic details of the pinning are still not fully understood.

We attempt to shed some light on the microscopic interaction between vortices and columnar defects by establishing a one-dimensional model based on the Ginzburg-Landau equations expressed in cylindrical coordinates. Numerical integration of the boundary value problem yields a detailed description of the behavior of the fields, order parameter and supercurrents near the defect, and thus a more fundamental scheme for calculating the pinning energy. This pinning energy can then be used to estimate from first principles certain thermodynamic properties of the vortex state. In addition, the understanding of the microscopic vortex-column interaction provides information for optimizing the pinning of vortices by choosing proper characteristics of the columnar defects.

\section{METHOD AND JUSTIFICATIONS}

Let us first consider a triangular array of vortices on a triangular array of columnar defects with the magnetic field parallel to the defects. We may approximate the hexagonal cells surrounding a vortex as circles, and solve the GinzburgLandau equations ${ }^{5}$ by assuming cylindrical symmetry, as shown in Fig. 1. The area $S$ of each cell is determined by the applied field $H_{a}$, such that $S=\Phi_{0} / H_{a}$, where $\Phi_{0}$ is the flux quantum. So far as this approximation is valid, we would expect all the results in this paper to remain accurate in the limit of triangular lattices of vortices and defects. That is, vortices will both be pinned and at the same time be able to adjust themselves into a regular lattice through the vortexvortex interaction. Thus we solve the Ginzburg-Landau equations by approximating the effect of nearby vortices through a cylindrical shell with symmetrical boundary conditions on the periphery.

Although the case of a perfect triangular vortex lattice (for which our calculations will be most accurate) can only be maintained under very special conditions, we remark that these conditions may be realized in the near future if artificially created triangular lattices of defects are developed in thin film superconductors. Similar concepts of artificially created defects have been demonstrated in low-temperature superconductors with square lattices of defects. ${ }^{6}$

We also speculate that the assumption of a cylindrical symmetry remains reasonably accurate even under conditions of reduced symmetry - that is, in the case of random but uniform distributions of parallel columnar defects and vortices. We assume that on average, the pinning of any given vortex will not depend on the actual position of its neighboring vortices. This is not unreasonable for several reasons. First, the symmetry is only broken near the boundary of the circular cell, not near the vortex core. Therefore the approximation is still valid as long as the vortex core and the defect size are much smaller than the size of the cell. That is, if $\xi(T), a \ll d$, where $\xi(T)$ is the superconducting coherence length, $a$ the defect radius and $d=\sqrt{\Phi_{0} / \pi H_{a}}$. Also, our assumption depends mainly on the orientational order, which, unlike the translational order, has been shown to be insensitive to disorder. ${ }^{7-10}$ The vortex-vortex interac-

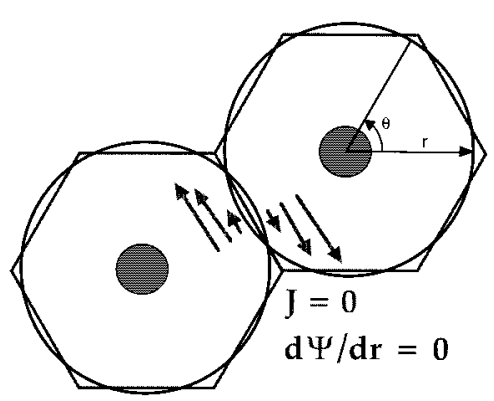

FIG. 1. Sketch of two cells within each of which the GinzburgLandau equations are integrated under the assumption of cylindrical symmetry. The presence of neighboring vortices enforces two outer boundary conditions $d f / d z=0$ and $J=0$, while the presence of the defect at the center of the cell provides two inner boundary conditions.

15372 C 1996 The American Physical Society 
tions rearrange vortices into a short range order within the "Larkin domain" (Refs. 11 and 12) in the presence of pinning defects, and from vortex imaging, it is found that vortices are almost always surrounded by five, six or seven nearest neighbors. ${ }^{13}$ Even if a few of them may be closer or farther than the others, the vortex-vortex interactions in a reasonably large magnetic field $\left(H_{a} \gg B_{\phi}\right)$ are strong enough that on the average the solutions will not deviate much from the ideal cylindrically symmetric solutions we envision. Finally, as the field strength decreases well below $B_{\phi}$, the vortex-defect interaction energy will dominate over the vortex-vortex interaction, thereby rendering the exact treatment of the neighboring vortices insignificant. In particular, we shall prove in the following calculations that the strength of the pinning energy is nearly independent of the strength of the magnetic field as long as the spacing between vortices is much larger than the defect radius. This suggests that since pinning does not depend on the vortex-vortex separation, it does not vary with small variations in the positions of the nearest neighbors.

However, we caution that the validity of the assumption of cylindrical symmetry must not be overexaggerated. If the defect distribution is random, there will be situations in which the vortex-vortex interactions will cause individual vortices to favor positions other than the defect sites. This effect will be most severe if the number of vortices is comparable to the number of defects $\left(H_{a} \sim B_{\phi}\right)$. Therefore the validity of our approach should be restricted to either vortex systems interacting with regular arrays of columnar defects, or those with random columnar defects and in the limits of either $H_{a} \ll B_{\phi}$ or $H_{a} \gg B_{\phi}$.

Assuming the validity of circular cells under the conditions described above, we choose the center of a vortex as the origin, and consider the variations of the free energy as the cylindrical defect is placed at the origin, or at the periphery of the cell where it will not affect the distribution of the order parameter and the supercurrents of the central vortex in an important way. The boundary conditions at the boundary of the defect are those given by de Gennes ${ }^{14}$ depending on whether the defect region is insulating or conducting.

The resulting energy difference indicates whether placing a vortex inside a defect is more favorable than placing the vortex as far as possible from the defect. We take a positive difference to imply favorable pinning, and call it the pinning energy. Equating this difference to the depth of the pinning potential, we then make use of the vortex/two-dimensional boson analogy $y^{3,4}$ to calculate the depinning temperature.

\section{A. Equations}

We shall solve the Ginzburg-Landau equations for a superconductor: ${ }^{5}$

$$
\begin{gathered}
\left(\frac{\nabla}{i}-\frac{2 \pi \mathbf{A}}{\Phi_{0}}\right)^{2} \Psi=\frac{1}{\xi^{2}} \Psi\left(1-\frac{|\Psi|^{2}}{\left|\Psi_{\infty}\right|^{2}}\right) ; \\
\mathbf{J}=\frac{c}{4 \pi} \nabla \times \nabla \times \mathbf{A}=\frac{e^{*}}{m^{*}}|\Psi|^{2}\left(\hbar \nabla \phi-\frac{e^{*}}{c} \mathbf{A}\right) ;
\end{gathered}
$$

where $\mathbf{A}$ is the vector potential, $\Phi_{0}$ the flux quantum, $\Psi$ the order parameter, $\Psi_{\infty}$ the order parameter in an infinite sample, $\xi$ the coherence length, and $e^{*}$ and $m^{*}$ the Cooper- pair charge and mass, respectively, while $\phi$ denotes the phase of the order parameter and $\mathbf{J}$ represents the current density.

Placing a vortex line at the origin, and assuming cylindrical symmetry, so that the phase $\phi$ varies as an integer multiple of the angle $\theta$ and all other quantities depend only on $r$, we have $\Psi=\left|\Psi_{\infty}\right| f(r) e^{i n \theta} \quad$ and $\quad \mathbf{A}=A(r) \hat{\theta} \quad$ with $A(r)=(1 / r) \int_{0}^{r} r^{\prime} H\left(r^{\prime}\right) d r^{\prime}$, where $H$ is the magnetic field, and $(r, \theta)$ are the polar coordinates in the cylindrical cell ${ }^{5}$ as shown in Fig. 1. Thus $n$ denotes the number of flux quanta located at the origin. The equations now become

$$
\begin{gathered}
-\frac{d^{2} f}{d r^{2}}-\frac{1}{r} \frac{d f}{d r}+\left(\frac{n}{r}-\frac{2 \pi A}{\Phi_{0}}\right)^{2} f=\frac{1}{\xi^{2}} f\left(1-f^{2}\right) ; \\
-\frac{d^{2} A}{d r^{2}}-\frac{1}{r} \frac{d A}{d r}+\frac{A}{r^{2}}=\frac{\Phi_{0}}{2 \pi \lambda^{2}} f^{2}\left(\frac{n}{r}-\frac{2 \pi A}{\Phi_{0}}\right) ;
\end{gathered}
$$

where we have used the relation $\lambda^{2}=m^{*} c^{2} / 4 \pi e^{* 2}\left|\Psi_{\infty}\right|^{2}$ for the penetration depth. In addition to the Ginzburg-Landau parameter $\kappa=\lambda / \xi$, like Abrikosov we define the dimensionless quantities $z=r / \lambda$ and $Q=2 \pi \xi A / \Phi_{0}-n / z \kappa,{ }^{15}$ so that Eqs. (3) and (4) can be expressed in terms of $f(z)$ and $Q(z)$ by the following equations:

$$
-\frac{1}{\kappa^{2}}\left(\frac{d^{2} f}{d z^{2}}+\frac{1}{z} \frac{d f}{d z}\right)+Q^{2} f=f\left(1-f^{2}\right)
$$

and

$$
\frac{d^{2} Q}{d z^{2}}+\frac{1}{z} \frac{d Q}{d z}-\frac{Q}{z^{2}}=f^{2} Q
$$

For later reference, we note that after the transformation the magnetic field is now measured in units of $\sqrt{2} \mathrm{H}_{c}$, while energy is measured in units of $\left(H_{c}^{2} / 4 \pi\right) \lambda^{3}$, where $H_{c}$ is the thermodynamic critical field.

The use of the dimensionless quantities reduces the problem to only two parameters: the defect radius $a$ and the applied magnetic field $H_{a}$, both of which affect the problem only through the boundary conditions. The third parameter, $\kappa$, is purely dependent on the properties of the material under consideration.

\section{B. Boundary conditions}

We will integrate Eqs. (5) and (6) from the origin to a distance of about one-half the typical separation of the vortices at a given magnetic field $H_{a}$. That is, we will be integrating out to a distance given by $d=\sqrt{n \Phi_{0} / \pi H_{a}}$, or $d$ $=\sqrt{2 n / \kappa H_{a}}$ in terms of the dimensionless units. In most cases, we will consider one flux quantum at a time, i.e., $n=1$. However, for large defect radii, it may become energetically favorable to have two flux quanta trapped in a single defect-in that case we will use $n=2$.

The magnetic field thus imposes an important boundary condition that restricts the length scale of the variation in the current density. As the currents are forced into a smaller region with increasing field, they become less effective at shielding the magnetic field that ultimately permeates the whole superconductor. We shall only concern ourselves with extreme type-II superconductors at applied fields $H_{a}$ much 
larger than the lower critical field $H_{c_{1}}$, so that the magnetic field profile is practically constant throughout the sample.

We also define the average areal density of columnar defects by $n_{\phi}=\Phi_{0} / B_{\phi}$, where $B_{\phi}$ is the matching field. ${ }^{1,2}$ By solving the equations within a cell defined by a single vortex and a single column, we obtain results for $B=B_{\phi}$. However, we will extend the calculations to the cases $B<B_{\phi}$ and $B>B_{\phi}$ later.

Next let us consider the boundary conditions themselves. At the outer boundary, based on symmetry considerations as shown in Fig. 1, we approximate the presence of the other vortices by requiring that the derivative of the order parameter be zero. We also want the currents to vanish, so that

$$
\frac{d f}{d z}=0
$$

and

$$
J=-\frac{3}{2} \sqrt{3} f^{2} Q J_{0}=0,
$$

where $J_{0}$ is the depairing current density. Equations (7) and (8) are the two boundary conditions for the outer boundary of the cell. Their physical significance is to average the effect of the nearest-neighbor vortices. Thus, for higher fields, the pinning energy we calculate will reflect the difference in the interaction between two trapped vortices and two free vortices. We also remark that the boundary condition given by Eq. (7) is in fact valid over a significant range of $z(\equiv r / \lambda)$, so long as the condition $d \gg a, \xi$ is satisfied, as illustrated in Fig. 2 which will be described in more details later. Consequently, even if the vortex distribution is neither perfect nor completely random, so that the outer boundary is not perfectly circular, we can always find an outer periphery where both Eqs. (7) and (8) are valid.

Next, we consider the inner boundary conditions. If we solve for a free vortex line, that is, a vortex line outside a columnar defect, we use the asymptotic expression for the order parameter given by Abrikosov ${ }^{15}$ to integrate out of the singularity at $z=0$ in Eqs. (5) and (6). For small $z, f=C z$ and $Q=1 /(\kappa z)^{D}$, where $C$ and $D$ are two parameters to be determined when solving the boundary value problem.

On the other hand, if the vortex line sits in a columnar defect, we at first take the boundary condition given by de Gennes for a bulk superconductor, bulk normal metal interface: ${ }^{14}$

$$
\left.\left(\frac{\hbar}{i} \nabla-\frac{e^{*}}{c} \mathbf{A}\right) \Psi\right|_{n}=\frac{1}{b} \Psi .
$$

Here $1 / b$ is a real proportionality constant and has the dimensions of inverse distance. In our case, Eq. (9) constrains the radial derivative of the order parameter $f$ on the boundary of the defect.

As shown by de Gennes ${ }^{14}$ for a superconductor/insulator (or vacuum) interface, the parameter $1 / b$ can be taken to be zero, while for a pure metal, $b \sim \hbar v_{F N} / k_{B} T$, where $v_{F N}$ is the electron velocity at the Fermi energy. While the temperature dependence of the boundary condition can be neglected for insulating defects, it must be taken into account for metallic defects. Specifically, for the metallic defects, $\lambda / b \rightarrow 0$ as
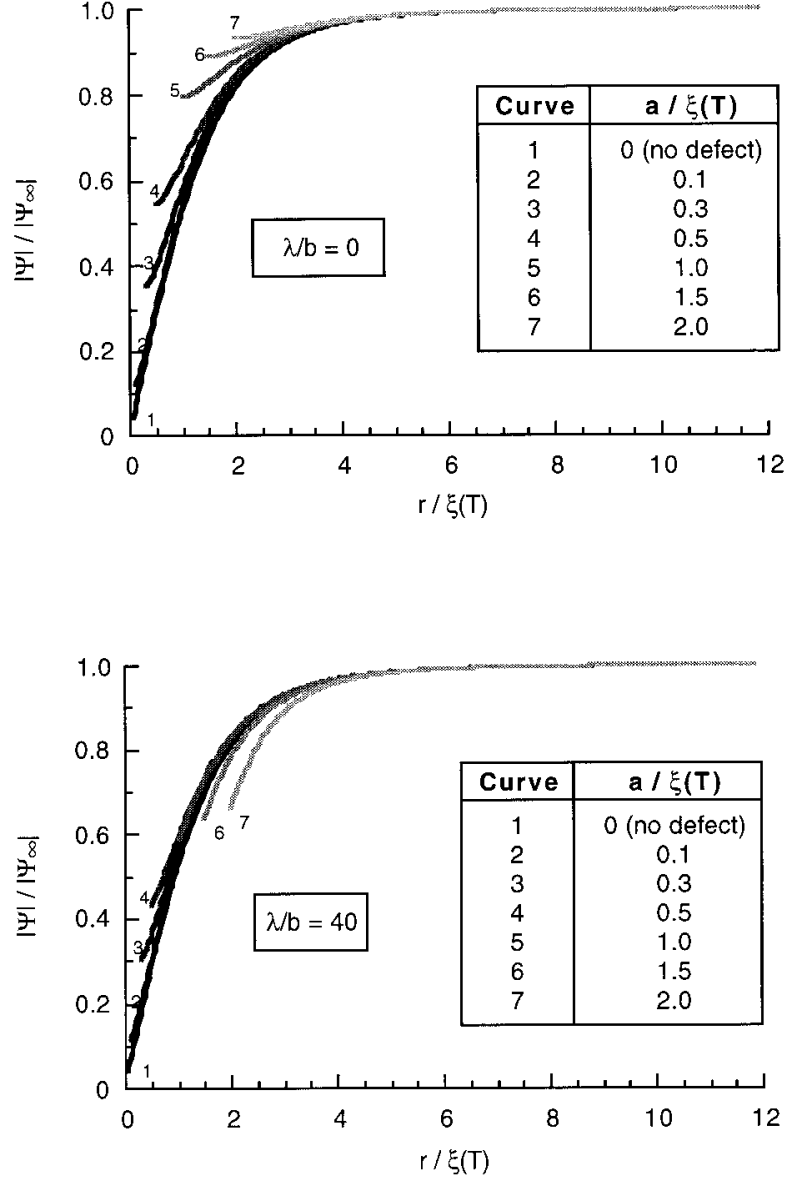

FIG. 2. Order parameter versus position for different defect radii, for insulating defects $(\lambda / b=0)$ and metallic defects $(\lambda / b=40)$. The magnetic field is $H_{a}=\sqrt{2} H_{c}$, and our calculations only consider the values of $|\Psi|$ in the range of $r \geqslant a$. In this paper, we always use $\kappa=70$.

$T \rightarrow 0$, and $\lambda / b \rightarrow \infty$ as $T \rightarrow T_{c}$. At $T=0.9 T_{c}$, we expect $\lambda / b \sim 40$ for a material with a Fermi velocity of the order of $1.2 \times 10^{7} \mathrm{~cm} / \mathrm{s}$. Our calculations, which are to be presented in the next section, show that the pinning energy is significantly altered by such a boundary condition in the case of defect radii $a>\xi$.

It should be noted that the boundary condition given by Eq. (9) may be inadequate for small defects that can hardly be considered as forming a bulk material. Thus the validity of this boundary condition may be severely limited for small defect radii. For instance, one can imagine possible Josephson tunneling effects when the defect size becomes much smaller than the superconducting coherence length. In metallic defects, there may be important proximity effects. The correct procedure will require solving the self-consistent Bogolubov equations ${ }^{14}$ in the vicinity of the defect, which is beyond the scope of our current approach.

In addition to the inner boundary condition given by Eq. (9) for the order parameter, we still need a second boundary condition for the magnetic field. Like Douglass in the treatment of a thin cylindrical shell, ${ }^{16}$ we assume that the magnetic field profile is constant inside the defect, so that at the boundary of the column $A=z(d A / d z)$.

Given the above four boundary conditions (two at the center of the cell and two at the periphery of the cell), we 
proceed to integrate the Ginzburg-Landau equations using a fifth-order, variable step-size Runge-Kutta algorithm, as presented in Ref. 17. The boundary value problem is solved using a customized shooting algorithm that seeks only the physically relevant solutions.

\section{Calculation of the pinning energy}

In order to compute the pinning energy of a vortex by a columnar defect, we need to consider the Gibbs free energy density of a superconductor, either with or without columnar defects, in the presence of vortex arrays. The Gibbs free energy density ${ }^{5}$ is given by

$$
\begin{aligned}
G_{S H}= & G_{N H}+\frac{H_{c}^{2}}{8 \pi}\left(f^{4}-2 f^{2}+2 \xi^{2} \mid \nabla\left(f e^{i n \phi}\right)\right. \\
& \left.-\left.\frac{i e^{*}}{\hbar c} f e^{i n \phi} A\right|^{2}\right)+\frac{\left[H(r)-H_{a}\right]^{2}}{8 \pi},
\end{aligned}
$$

so that after conversion to our dimensionless quantities and integration over a lattice cell we obtain the line energy for a vortex:

$$
\begin{gathered}
\frac{\pi a^{2}\left[H(a)-H_{a}\right]^{2}}{8 \pi}+2 \pi \int_{a}^{d}\left[\frac { 1 } { 1 6 \pi } \left(f^{4}-2 f^{2}\right.\right. \\
\left.\left.+2\left(\frac{f^{\prime 2}}{\kappa^{2}}+Q^{2} f^{2}\right)\right)+\frac{\left[H(z)-H_{a}\right]^{2}}{8 \pi}\right] z d z,
\end{gathered}
$$

where $a$ is the radius of the columnar defect. Next, we calculate the pinning energy by taking the difference between the energy of the vortex sitting outside the defect and the vortex sitting inside the defect. In the second case, we correct for the loss of condensation energy resulting from the presence of the defect. The correction in the line energy is given by $\Delta f=\left(f^{4}-2 f^{2}\right) \pi a^{2} / 16 \pi$ for every unoccupied defect.

If $B<B_{\phi}$ (i.e., there are fewer vortices than cylindrical defects), we assume that the pinning energy per vortex remains the same as in the case of $B=B_{\phi}$ on a regular array of defects. This is justified by the fact the additional defects below the matching field will remain unoccupied by vortices; therefore, their contribution to the individual pinning energy of single vortex-column pairs will be negligible. Furthermore, the vortex-vortex interaction will remain important enough for the vortex lattice to rearrange itself so that the assumed cylindrical symmetry will remain adequate. The same argument remains valid in the case of a random distribution of defects, provided $B \ll B_{\phi}$.

If $B>B_{\phi}$, there are fewer defects than vortices, and unless the defects are large enough to support multiple flux quanta, some vortices will remain unpinned at all times. In that case, we can still calculate an average energy density over the sample, with some vortices in a defect and some vortices free. This energy density can be compared to the average energy density for $n=1$ vortices and $n=2$ vortices, to determine whether it is energetically favorable for a defect to trap more than one vortex. Again, for a random distribution of defects, the results will only be valid for $B \gg B_{\phi}$.

\section{Calculation of the depinning temperature}

To compare our calculations with relevant physical quantities, such as the depinning temperature (temperature at which the vortices "escape" from the columnar defects because of thermal motion), we follow the approach given by Nelson and Vinokur, ${ }^{3}$ which maps the problem of a vortex trapped in a columnar defect to the problem of a twodimensional Boson in a potential well. That is, we may set the mass $m$ of the Boson equal to the vortex line energy $\epsilon_{0}$, $\hbar$ equal to the temperature $T$, and the depth of the potential well equal to the pinning energy per unit length $\epsilon_{r}$ calculated as described in the previous section and converted to the Gaussian units. Thus the vortex problem becomes a standard quantum mechanical one, which can be solved and mapped back to give an effective temperature-dependent pinning potential $\epsilon_{r}(T) \simeq \epsilon_{r} f[T / \beta(T)]$, where the exact forms of $f(x)$ and of $\beta$ depend on the details of the model, ${ }^{3,4}$ such as the shape assumed for the potential well. Here $\epsilon_{r}$ is the pinning energy at $T=0$, while $\epsilon_{r}(T)$ is the pinning energy renormalized due to thermal fluctuations. The solution to the selfconsistency equation $T_{\mathrm{dp}}^{r}=\beta\left(T_{\mathrm{dp}}^{r}\right)$ is defined as the depinning temperature, at which $f(x)$ and therefore $\epsilon_{r}(T)$ rapidly drop to zero. Assuming a potential well with a radial dependence of the pinning energy given by $\epsilon_{r}(R)$ $=\epsilon_{r} /\left(R^{2} / 2 \xi^{2}+1\right)$ (this approximation is valid for large $R$ ), we obtain ${ }^{3,4}$

$$
T_{\mathrm{dp}}^{r}=\frac{2 \xi\left(T_{\mathrm{dp}}\right)}{\pi} \sqrt{\epsilon_{l} \epsilon_{r}}
$$

where $\epsilon_{l}=\left[\Phi_{0} / 4 \pi \lambda\left(T_{\mathrm{dp}}\right)\right]^{2} \ln \kappa$ is the line tension. The pinning energy per unit length is given by

$$
\epsilon_{r}\left(T, a, H_{a}\right)=\frac{H_{c}^{2}(T)}{4 \pi} \lambda^{2}(T) e_{p}\left(T, a, H_{a}\right),
$$

where $e_{p}$ is the pinning energy per unit length in our dimensionless units. For a given value of $\kappa, e_{p}$ is a function of the defect radius $a$ measured in terms of the penetration depth $\lambda$, as well as of the applied field $H_{a}$ measured in terms of $\sqrt{2} H_{c}$. Thus the pinning energy depends on temperature: $e_{p}\left(T, a, H_{a}\right)=e_{p}\left[a(T), H_{a}(T)\right]$, where $a(T)$ denotes the effective defect size at a finite temperature $T$ in our dimensionless calculations. Similarly, $H_{a}(T)$ denotes the effective applied magnetic field strength. To incorporate the temperature dependence into our calculations within a fixed cell, we note that the penetration depth can be approximated by $\lambda(T)$ $\approx \lambda(0) / \sqrt{1-T / T_{c}}$. More accurate models for the temperature dependence of the penetration depth could also be used. Thus the effective defect size decreases as temperature increases. More precisely, we have $a(T) \approx a(0) \sqrt{1-T / T_{c}}$. Since $H_{c}(T) \approx H_{c}(0)\left(1-T / T_{c}\right)$, we also obtain an effective applied field $H_{a}(T) \approx H_{a}(0) /\left(1-T / T_{c}\right)$. As temperature increases, the effective magnetic field increases. Note that the integration cell radius decreases at the same rate as the effective defect size. The pinning energy per unit length becomes

$$
\epsilon_{r}\left(T, a, H_{a}\right)=\frac{\Phi_{0}^{2}}{32 \pi^{3} \xi^{2}(0)}\left(1-\frac{T}{T_{c}}\right) e_{p}\left[a(T), H_{a}(T)\right]
$$


Inserting Eq. (14) into the implicit equation for the depinning temperature, we finally obtain

$$
T_{\mathrm{dp}}=\frac{\Phi_{0}^{2}}{(2 \pi)^{7 / 2}} \frac{\sqrt{\ln \kappa}}{\lambda(0)} \sqrt{1-T_{\mathrm{dp}} / T_{c}} \sqrt{e_{p}\left[a\left(T_{\mathrm{dp}}\right), H_{a}\left(T_{\mathrm{dp}}\right)\right]} .
$$

The solution of Eq. (15) must be determined numerically.

For metallic defects, the boundary conditions themselves are temperature dependent. In that case, the calculation becomes slightly more complicated since we now have $e_{p}\left(T, a, H_{a}, \lambda / b\right)=e_{p}\left[a(T), H_{a}(T), \lambda(T) / b(T)\right]$. However, the overall procedure is consistent with that given by Eqs. (14) and (15). Also, as far as simple numerical estimates are concerned, the depinning temperature will depend only on the pinning energy for small defect radii $a<\xi$, at which the pinning energy is independent of the applied magnetic field $H_{a}$. Neglecting the field dependence greatly simplifies the calculations, both for metallic and for insulating defects.

\section{RESULTS AND DISCUSSION}

\section{A. Behavior of the order parameter, fields, and currents}

The spatial dependence of the order parameter for different radii and conductivities of the columnar defects is shown in Fig. 2. The curves as plotted in Figs. 2 and 3 are the solutions to the Ginzburg-Landau equations - we did not attempt to draw the behavior of the order parameter inside the defects. Thus the curves begin at the boundary of the defect, and end at the separation between two neighboring cells. Inside insulating defects, we would expect the order parameter to drop very rapidly to zero. Inside metallic defects, we might expect a proximity effect. However, these considerations are beyond the scope of this paper.

We note that a larger value of the proportionality constant $\lambda / b$, which corresponds to a higher conductivity of the columnar defect, lowers the order parameter near the boundary. Similarly, the magnitude of the order parameter also decreases near the boundary as the defect radius decreases. As the defect radius goes to zero, the solutions approach those for a free vortex, as expected. It is also interesting to compare the magnitude of the order parameter near the boundary of a pinned vortex with that for a free vortex. At a given position $z$ outside a sufficiently large defect, the magnitude of the order parameter is larger in the case of insulating defects $(\lambda / b=0)$ than in the case of metallic defects $(\lambda / b$ =40). This difference can be interpreted as follows: For insulating defects, the order parameter accumulates outside the defect, whereas the order parameter "leaks" into metallic defects through the proximity effect, thereby depleting the order parameter in the vicinity of the defect.

Next, we consider the current distribution near a columnar defect. As the defect size decreases, the magnitude of the currents near the interface (Fig. 3) at first increases, passing the depairing current density $J_{0}=c H_{c} / 3 \sqrt{6} \pi \lambda$ for $a \leqslant 2 \xi$ in the case of insulating defects. This result at first glance seems troubling because Cooper pairs cannot support currents beyond the depairing current density. However, if we take into account the abnormal increase of the order parameter near the periphery of the insulating defect, and the possibility of enhancement of superconductivity near the inter-
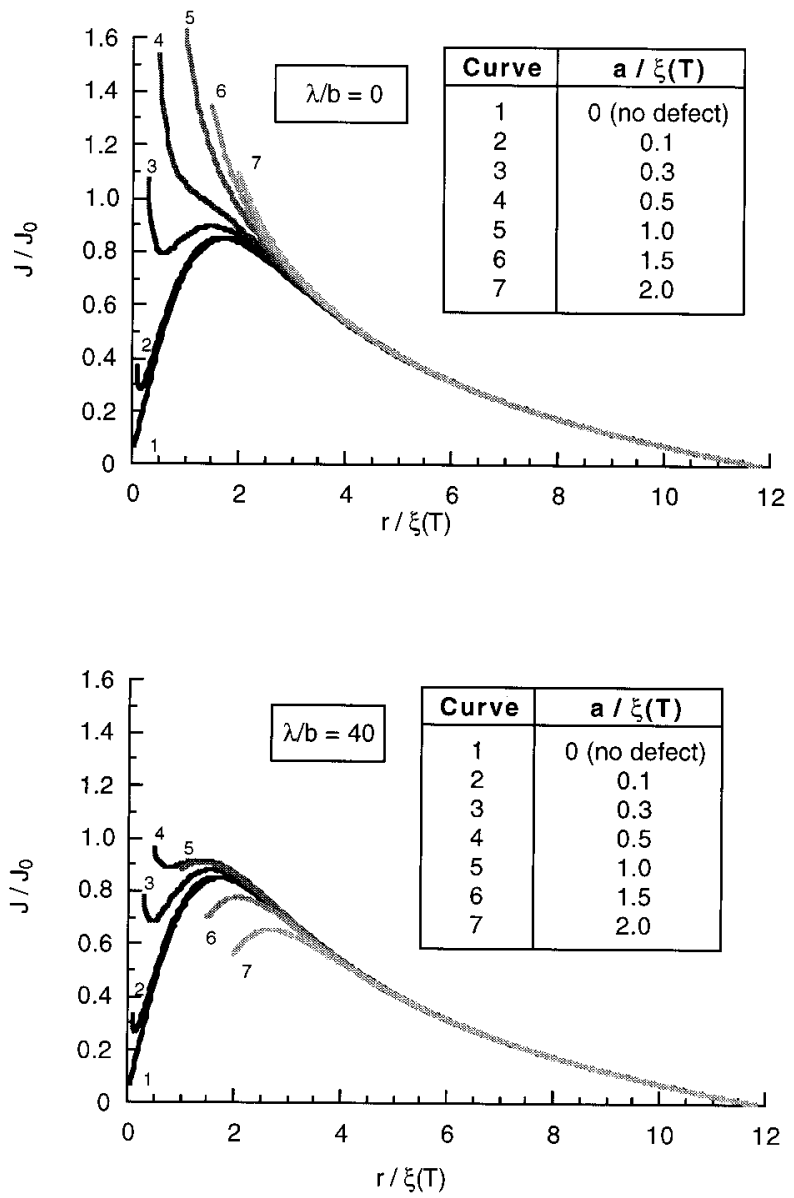

FIG. 3. Supercurrent density versus position for different defect radii, for insulating defects $(\lambda / b=0)$ and metallic defects $(\lambda / b=40)$, with $H_{a}=\sqrt{2} H_{c}$ and $\kappa=70$. The calculations are only performed for $r \geqslant a$.

face, which is analogous to the existence of a surface critical field $H_{c_{3}} \simeq 1.7 H_{c_{2}}$ for magnetic fields parallel to the superconductor/insulator interface, ${ }^{14}$ it is not unreasonable that the current may exceed $J_{0}$ near the insulating defect. In contrast, we note that the currents are always smaller than $J_{0}$ for metallic defects $(\lambda / b \simeq 40)$.

As the defect size decreases further, the currents develop a local maximum slightly below the depairing current density, and approach the current distribution for a free vortex. It is interesting to note that in this latter range, the current distribution also has a local minimum; the vortex adopts a two-layered structure. It remains to be seen whether this behavior is true to reality, or whether it is an artifact due to the approximations involved in determining the boundary conditions. Spectroscopy studies using a low-temperature scanning tunneling microscope may provide better insights into this issue.

\section{B. Pinning energy}

The dependence of the pinning energy on the defect radius is shown in Fig. 4 for various applied magnetic fields. The calculations assume that $B \leqslant B_{\phi}$ for all fields $\left(B \ll B_{\phi}\right.$ for irregular distributions of defects). However, we can assume that the pinning energy of an individual vortex will not be 

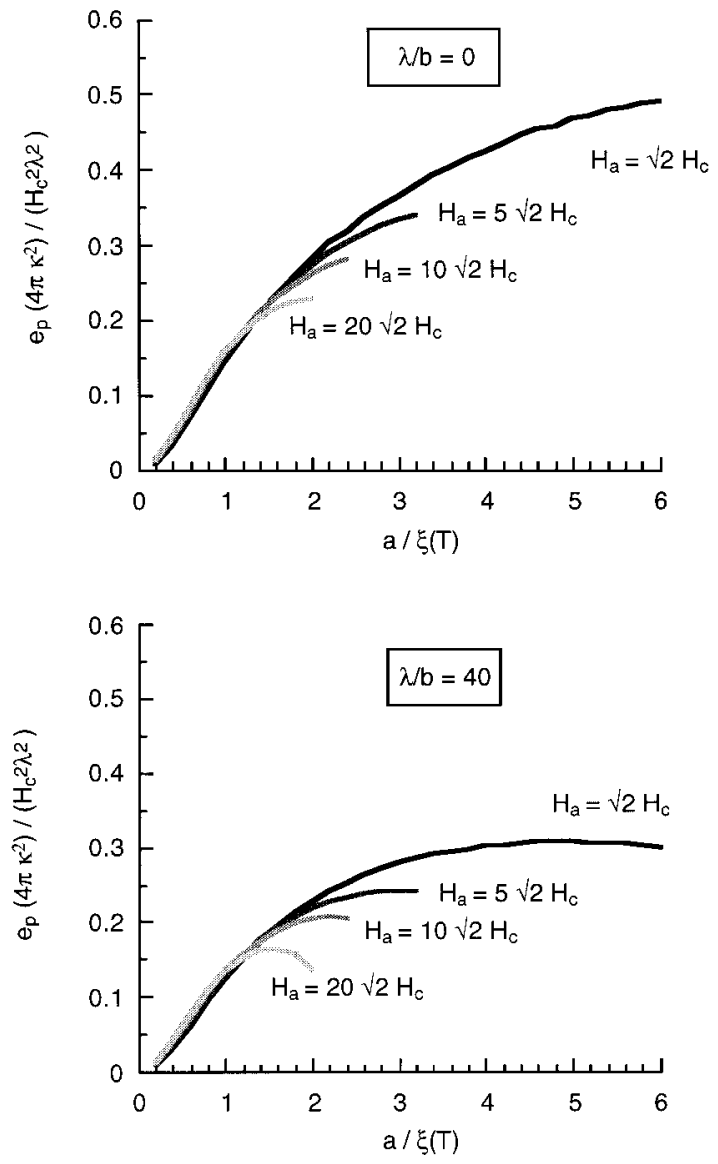

FIG. 4. Pinning energy as a function of defect size at four different applied fields $H_{a}$, for insulating defects $(\lambda / b=0)$ and metallic defects $(\lambda / b=40)$. The curves are discontinued at higher magnetic fields for which the model becomes inaccurate.

modified for $B>B_{\phi}$. Since not every vortex is pinned at these fields, the pinning energy in fact only applies to a fraction of the vortices.

One important feature of the result is that the pinning energy decreases with the increasing field if the defect radius is sufficiently larger than $\xi$. The pinning energy becomes independent of the applied field strength if $a<\xi$. However, due to the approximations in the model, we shall not overinterpret the correctness of the results for defect radii larger than about half the cell radius. This is why the curves in Fig. 4 are truncated.

The direct comparison to other calculations of the pinning energy is difficult because of the incompatibilities in the assumptions. For example, Mkrtchyan and $\mathrm{Schmidt}^{18}$ calculate the pinning energy of a vortex in a single cylindrical defect in the London limit. They assume much larger defect sizes $(a \gg \xi)$ and much lower magnetic fields, so that $d \gg \lambda$. They obtain an expression for the pinning energy of $e_{p}=\left(1 / 4 \kappa^{2}\right) \ln (\kappa a / 2)$, valid only for $a \gg \xi$. While the magnitude of the pinning energy is comparable to the one we obtained, their calculations take into account neither the variation of the order parameter nor the close proximity of other vortices. These two contributions clearly dominate the pinning for small $(a \simeq \xi)$ defect radii.

For very small defects $(a<\xi)$, we obtain a pinning energy about three times larger than the estimate given by Blatter

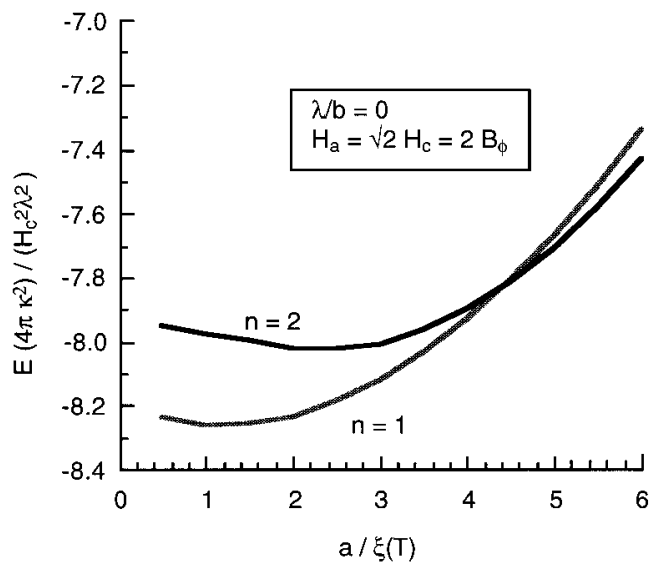

FIG. 5. Average free energy per vortex (and per unit length) at twice the matching field $\left(H_{a}=\sqrt{2} H_{c}\right)$ for insulating defects $(\lambda / b$ $=0)$. On the $n=1$ curve, each defect is occupied by exactly one vortex. All additional vortices are free. In the $n=2$ case, each defect is occupied by two vortices-all vortices are pinned. Note that both the vertical and horizontal scales are temperature dependent. Placing two vortices in the same defect becomes favorable for large defect radii (4 to 5 coherence lengths).

et $a .^{3}$ both for insulating defects and for metallic defects. (In the reduced units, they estimate $e_{p} \approx a^{2} / 16$, where $a$ is measured in units of $\lambda$.) Since the differences in the pinning energy due to the conductivity of the columnar defect only appear if the defect radii are larger than those of the vortices, a larger value of the temperature dependent parameter $\lambda / b$ does not resolve the discrepancy in the case of $a<\xi$. This implies that the change in the behavior of the order parameter is not the only cause of the pinning; for small defects, the supercurrent contribution to the pinning energy is at least as significant: We have indeed verified that the contribution of supercurrents to the total energy does account for this difference. On the other hand, for $a>\xi$, Blatter $e t a l .{ }^{3}$ use Mkrtchyan and Schmidt's results, ${ }^{18}$ and therefore the same limitations discussed in the previous paragraph apply.

For larger defects, the possibility of multiple flux quanta in each defect exists. However, at or below the matching field, it is never advantageous to place more than one flux quanta in a defect. For higher magnetic fields, it will be energetically favorable to place two vortices in a single defect, rather than leave one of the vortices outside the defect, provided the defect radius is approximately 4 to 5 times the coherence length (Fig. 5). Note that according to Mkrtchyan and Schmidt, ${ }^{18}$ entrapment of a second vortex becomes favorable only for much larger defects (for which the defect size is at least $0.3 \lambda$ ). We believe that the difference between our results and those by Mkrtchyan and Schmidt is in the consideration of neighboring vortices. We have implicitly included the presence of all neighboring vortices into the boundary conditions, whereas Mkrtchyan and Schmidt only considered the interaction between a defect and one additional free vortex in an infinite sample with no other vortices present.

\section{Depinning temperature}

Using the method described above in Sec. II D, and the numerical values typical for $\mathrm{YBa}_{2} \mathrm{Cu}_{3} \mathrm{O}_{7}$ of $\xi(0)=2 \mathrm{~nm}, \lambda(0)$ $=140 \mathrm{~nm}, T_{c}=93 \mathrm{~K}$, we obtain the depinning temperatures $T_{\mathrm{dp}}$ in Fig. 6, in the case of insulating defects. Note that the 


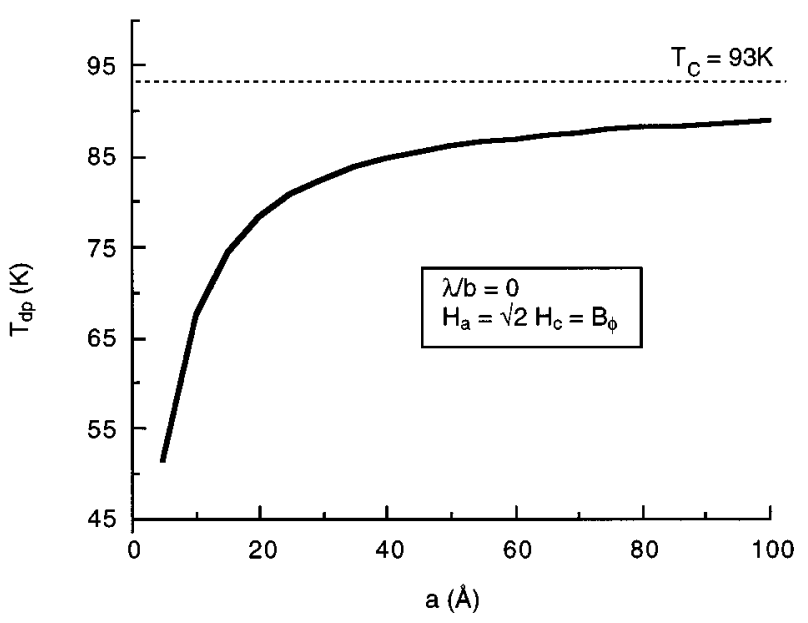

FIG. 6. Depinning temperature versus defect radius for $\lambda / b=0$, with parameters typical of $\mathrm{YBa}_{2} \mathrm{Cu}_{3} \mathrm{O}_{7}: \xi(0)=2 \mathrm{~nm}, \lambda(0)=140$ $\mathrm{nm}, \kappa=70$, and $T_{c}=93 \mathrm{~K}$. The model is not accurate enough to calculate the magnetic field dependence of the depinning temperature.

temperatures are only accurate to within a few degrees, given the approximations used in the calculation. Also, our results appear to indicate a depinning temperature about $5 \mathrm{~K}$ higher than the one obtained by Blatter et al. ${ }^{4}$ However, the order of magnitude agreement is satisfactory.

For conducting defects, we found that for realistic values of the parameter $\lambda / b$ the depinning temperature is not lowered much with respect to that for insulating defects. It appears that the pinning is strong enough in both cases that depinning occurs only when $\xi(T) \gg a$, so that the differences in hole conductivity become insignificant in the case of hightemperature superconductors.

It is interesting to note the dependence of $T_{\mathrm{dp}}$ on the defect size. Once the radius of the defect is larger than about two coherence lengths, the depinning temperature levels off with a very slight increase towards the critical temperature. That is, as far as the depinning temperature is concerned, it is unnecessary for practical applications to create columnar defects with radii much larger than a few coherence lengths. At high temperatures, since the coherence length diverges, any defect size will quickly become negligible. However, this result might have to be modified if the exact shape of the potential well is taken into account.

\section{Comparison with other numerical calculations}

Finally, we compare the results in this work with other numerical calculations of vortex-pin interactions. ${ }^{19,20}$ Recent time-dependent Ginzburg-Landau calculations by Machida and $\mathrm{Kabasaki}^{20}$ have revealed interesting vortex dynamics in layered superconductors under the influence of point and columnar defects. However, in contrast to our approach which considers the vortex-pin interaction via direct solutions to a boundary value problem of an extreme type-II superconductor, Ref. 19 approximates the presence of columnar defects by taking a fixed defect radius $2 \xi(0)$, a small $\kappa=2$, and by assuming $T_{c}=0$ inside the defect. The calculations in Ref. 19 therefore do not provide direct information of the pinning potential for different sizes and conductivity of the columnar defects.
On the other hand, the two-dimensional time-dependent Ginzburg-Landau equations were recently solved by the research group at Argonne ${ }^{20,21}$ to study many-vortex dynamics in clean samples and along the grain boundaries. However, these calculations were also performed on a fairly coarse grid (with cells $\xi / 2$ wide) and with a value of $\kappa=4$, thereby not allowing an accurate determination of the variations of the order parameter and supercurrents in the vicinity of defects in the case of high- $T_{c}$ superconductors.

\section{CONCLUSION}

By solving the full Ginzburg-Landau equations, we have studied the microscopic effect of the defect size and conductivity on the variation of the order parameter and the supercurrents. The pinning energies and depinning temperatures for different defect sizes and conductivities have also been derived and compared to previous analytical estimates. We find that the defect size and conductivity have important consequences on the behavior of the order parameter and the supercurrents. However, their effect on the depinning temperature appears negligible, even though pinning itself is stronger for insulating defects than for metallic defects, at least for defect radii greater than the coherence length $(a>\xi)$.

We emphasize that the results are accurate only if the defect size $(a)$ and the coherence length $(\xi)$ are much smaller than the vortex-vortex separation $(a, \xi \ll d)$. The model should be valid for triangular lattices of vortices and defects. More speculatively, in the case of random distributions of defects, we expect the calculations to remain applicable provided that either $H_{a} \ll B_{\phi}$ or $H_{a} \gg B_{\phi}$.

There remain many issues to be addressed in future work: For example, a better self-consistent treatment of the inner boundary conditions will be necessary to provide more detailed understanding of the vortex-pin interaction. The current treatment of the boundary conditions, which is valid for bulk materials, may not be completely appropriate for the small geometries we are considering. The role of anisotropy also deserves attention. Finally, at a more challenging numerical level, it may be worthwhile to solve the GinzburgLandau equations in two dimensions to map out the actual shape of the potential well accurately for small radii. In addition, such calculations would allow a quantitative study of the effect of irregular vortex distributions, and thus lead to a much more accurate understanding of the pinning, and a much more accurate determination of the depinning temperature. Two-dimensional calculations may also allow the derivation of other important physical quantities such as the shear and compression elastic moduli for the vortex lattice.

\section{ACKNOWLEDGMENTS}

S.M. would like to acknowledge support from Caltech's Ph11 and SURF programs that made his participation possible. The work was supported in part by the National Science Foundation (Grants No. DMR94-01315 and No. DMR93-18931), the Office of Naval Research (Grant No. N00014-91-J-1556), and the David and Lucile Packard Foundation. 
${ }^{1}$ M. Konczykowski et al., Phys. Rev. B 44, 7167 (1991).

${ }^{2}$ L. Civale et al., Phys. Rev. Lett. 67, 648 (1991).

${ }^{3}$ N. R. Nelson and V. M. Vinokur, Phys. Rev. Lett. 68, 2398 (1992); Phys. Rev. B 48, 13060 (1993).

${ }^{4}$ G. Blatter et al., Rev. Mod. Phys. 66, 1327 (1994).

${ }^{5}$ M. Tinkham, Introduction to Superconductivity (Krieger, Malabar, 1975).

${ }^{6}$ M. Baert, V. V. Metlushko, R. Jonckheere, V. V. Moshchalkov, and Y. Bruynseraede, Phys. Rev. Lett. 74, 3269 (1995).

${ }^{7}$ H. F. Hess, R. B. Robinson, J. M. Valles, Jr., and J. V. Waszezak, Phys. Rev. Lett. 62, 214 (1989).

${ }^{8}$ Ch. Renner, A. D. Kent, Ph. Niedermann, O. Fischer, and F. Levy, Phys. Rev. Lett. 67, 1650 (1991).

${ }^{9}$ G. J. Dolan et al., Phys. Rev. Lett. 62, 2184 (1989).

${ }^{10}$ I. Maggio-Aprile, Ch. Renner, A. Erb, E. Walker, and O. Fischer, Phys. Rev. Lett. 75, 2754 (1995).

${ }^{11}$ A. I. Larkin, Sov. Phys. JETP 31, 784 (1970).

${ }^{12}$ A. I. Larkin and Yu. M. Ovchinnikov, Sov. Phys. JETP 38, 854 (1974); J. Low Temp. Phys. 34, 409 (1979).
${ }^{13}$ H. Dai, S. Yoon, J. Liu, R. C. Budhani, and C. M. Lieber, Science 265, 1552 (1994).

${ }^{14}$ P. G. de Gennes, Superconductivity of Metals and Alloys (Benjamin, New York, 1966).

${ }^{15}$ A. A. Abrikosov, JETP 32, 1442 (1957).

${ }^{16}$ D. H. Douglass, Jr., Phys. Rev. 132, 513 (1963).

${ }^{17}$ W. H. Press et al., Numerical Recipes in C (Cambridge University Press, Cambridge, 1992).

${ }^{18}$ G. S. Mkrtchyan and V. V. Shmidt, JETP 61, 367 (1971). Note that their expression for the pinning energy differs slightly from the value given by Blatter et al. in Ref. 4: $e_{p}$ $=1 / 4 k^{2} \ln (k a / \sqrt{2})$.

${ }^{19}$ M. Machida and H. Kabusaki, Phys. Rev. Lett. 75, 3178 (1995).

${ }^{20}$ I. Aranson, B. Ya. Shapiro, and V. M. Vinokur, Phys. Rev. Lett. 76, 1170 (1996).

${ }^{21}$ D. W. Braun, G. W. Crabtree, H. G. Kaper, A. E. Koshelev, G. K. Leaf, D. M. Levine, and V. M. Vinokur, Phys. Rev. Lett. 76, 831 (1996). 